\title{
USO DE MODELOS ESTATÍSTICOS PARA CLASSIFICAÇÃO DE SÍTIOS EM POVOAMENTOS DE Pinus taeda NA REGIÃO DE CAÇADOR - SC
}

\author{
Saulo Jorge Téo ${ }^{1}$, Diego Ricardo Bressan ${ }^{2}$, Reinaldo Hoinacki da Costa ${ }^{3}$ \\ ${ }^{1}$ Eng. Florestal, M.Sc., UNOESC, Xanxerê, SC, Brasil - sauloteo@ yahoo.com.br \\ ${ }^{2}$ Acadêmico de Engenharia Florestal, UNOESC, Xanxerê, SC, Brasil - d.bressan@ hotmail.com \\ ${ }^{3}$ Eng. Florestal, Juliana Florestal Ltda., Caçador, SC, Brasil - reinaldo@ frameport.com.br
}

Recebido para publicação: 25/01/2010 - Aceito para publicação: 28/06/2010

\begin{abstract}
Resumo
Este trabalho teve como objetivo testar diferentes modelos estatísticos para ajuste de curvas de índice de sítio e verificar se as curvas anamórficas geradas foram satisfatórias para classificação de sítios em povoamentos de Pinus taeda L. na região de Caçador, SC. Os dados de altura dominante $\left(\mathrm{h}_{\mathrm{dom}}\right)$ utilizados nesta pesquisa foram obtidos de parcelas permanentes de área fixa e formato retangular, com áreas de 500 e $625 \mathrm{~m}^{2}$. As parcelas foram distribuídas de forma aleatória nos povoamentos, a fim de abranger a maior variabilidade possível de produtividade. A seleção do melhor modelo estatístico ajustado foi feita por meio das seguintes estatísticas de ajuste e precisão: coeficiente de determinação ajustado $\left(\mathrm{R}_{\mathrm{aj}}{ }^{2}\right)$, erro padrão da estimativa (Syx), erro padrão da estimativa em porcentagem (Syx\%) e distribuição de resíduos em porcentagem. O modelo monomolecular apresentou os melhores resultados para os critérios de seleção, portanto foi escolhido para a construção das curvas de índice de sítio pelo método da curva guia. Depois de se proceder à classificação de sítios, foram realizados testes de anamorfismo, os quais indicaram o padrão anamórfico das curvas geradas pelo modelo estatístico ajustado para classificação de sítios dos povoamentos de Pinus taeda na região de Caçador, SC.

Palavras-chave: Modelos estatísticos; teste de anamorfismo; curvas de índice de sítio; altura dominante.
\end{abstract}

\begin{abstract}
Use of statistical modells for site classification of Pinus taeda plantation in the region of Caçador, Santa Catarina State, Brazil. This study aimed to test different statistical models for fitting site index curves and check if the anamorphic curves generated were satisfactory to classify the loblolly pine (Pinus taeda L.) plantations in the region of Caçador, Santa Catarina State, Brazil. The data of dominant height $\left(\mathrm{h}_{\mathrm{dom}}\right)$ used in this study were obtained from permanent plots of fixed area and rectangular shape, with areas of 500 and 625 square meters. The plots were distributed randomly in the stands to cover the largest possible variability of productivity. The selection of the best equation was made by the following fitting and precision statistics: adjusted coefficient of determination $\left(\mathrm{R}^{2}{ }_{\mathrm{aj}}\right)$, standard error of estimation (Syx), standard error of estimate in percentage (Syx\%) and graphical distribution of the residuals in percentage. The monomolecular model showed the best performance, so it was chosen for the construction of the site index curves by guide curve method. After construction of the site index curves, the stability of the curves was also tested, which indicated the anamorphic pattern of the curves generated by the equation for site classification of loblolly pine plantations in the region of Caçador, Santa Catarina State, Brazil.

Keywords: Statistical models; test of anamorphism; site index curves; dominant height.
\end{abstract}

\section{INTRODUÇÃO}

Os plantios de Pinus spp. no Brasil iniciaram-se há mais de um século. Entretanto o Pinus taeda L. foi introduzido somente por volta de 1948, em São Paulo, apresentando grandes vantagens, como facilidade nos tratos culturais e rápido crescimento das plantas. A partir disso, o potencial do gênero Pinus vem sendo explorado pelos investidores do meio florestal e melhorado para diversos fins, como produção de celulose de fibra longa, painéis reconstituídos, pasta de alto rendimento e madeira serrada, 
entre outros (SHIMIZU, 2008).

O Pinus taeda se adaptou muito bem aos sítios da região Sul, principalmente por ter boa resistência ao frio. Algumas procedências, como Carolina do Norte, por exemplo, apresentam grande tolerância a invernos rigorosos (SHIMIZU, 2008). Selle (1994) acrescentou que o Pinus taeda é uma das espécies mais plantadas e que a preferência pelo gênero se dá pelo crescimento rápido, alcançando grande incremento corrente anual.

Os sítios florestais são considerados como áreas homogêneas quanto às suas características ambientais (climáticas, edáficas, topográficas, biológicas etc.), apresentando, por sua vez, um desenvolvimento florestal correspondente ao potencial dessas características. As condições de sítio de um determinado local estão ligadas à capacidade produtiva deste.

Os ecologistas definem sítio como uma unidade geográfica uniforme, caracterizada por uma combinação estável dos fatores ambientais desse local, enquanto os técnicos em manejo florestal o definem como um fator de produção primário capaz de produzir madeira ou produtos florestais a ela associados (SHONAU, 1987, citado por MACHADO et al., 1997).

A maioria dos empreendimentos florestais ocupa vastas áreas de terra e apresenta características muito heterogêneas, resultando em diferentes níveis de produtividade. Manejar essas grandes áreas com floresta sem qualquer diferenciação ou classificação com relação ao seu potencial produtivo pode tornarse um processo com resultados insatisfatórios. Além disso, a classificação das florestas quanto à sua capacidade produtiva possibilita melhor avaliação quantitativa da floresta e a escolha de diferentes estratégias para melhorar a qualidade de cada sítio florestal.

Para classificar sítios existem métodos diretos e indiretos. Os métodos indiretos avaliam a qualidade do sítio a partir de atributos do ambiente, levando em consideração características como clima, solo e plantas indicadoras. Os métodos diretos utilizam basicamente indicadores da própria vegetação, que refletem as interações de todos esses fatores com o sítio (TONINI et al., 2001; SCOLFORO, 2006).

Segundo Scolforo (2006), entre os métodos diretos a altura dominante $\left(\mathrm{h}_{\mathrm{dom}}\right)$ é a variável dendrométrica que melhor se enquadra nos pré-requisitos para um bom indicador de sítio, que são: facilidade de determinação no campo, possuir boa correlação com a produção volumétrica, indicar a qualidade do sítio para todas as idades do povoamento e ser independente da densidade do povoamento.

A altura dominante de Assmann é muito utilizada para indicar a capacidade do sítio. O seu uso nessa classificação justifica-se por ser pouco influenciada pelos desbastes por baixo e por possuir alta correlação com a produtividade total do povoamento (FINGER, 2005).

Scolforo; Machado (1988a, 1988b), Tonini et al. (2001), Oliveira et al. (1998) e Selle et al. (1994) utilizaram $\mathrm{h}_{\mathrm{dom}}$ como indicador de qualidade de sítios para classificá-los em povoamentos de Pinus spp. no sul do Brasil.

O método da curva guia foi empregado por Machado et al. (1997) na região de Curitiba e por Batista; do Couto (1986) no estado de São Paulo para Mimosa scabrella e Eucalyptus spp., respectivamente. Em contrapartida, Scolforo (1992) utilizou o método da diferença algébrica para classificação de sítios em povoamentos de Pinus caribaea na região de Agudos, SP.

Dias et al. (2005) testou os métodos da curva guia, da equação das diferenças e da predição dos parâmetros para classificação de sítios em povoamentos de eucaliptos desbastados no município de Alagoinhas, Bahia. De acordo com os autores, os melhores resultados foram encontrados com o método da curva guia.

Dentre os modelos estatísticos ajustados por Scolforo; Machado (1988a, 1988b), Selle et al. (1994), Tonini et al. (2001) e Oliveira et al. (1998) para classificação de sítios em povoamentos de Pinus spp. na região Sul do Brasil, destacam-se os modelos de Chapman e Richards, Prodan, monomolecular e o modelo de Schumacher. Batista; do Couto (1986) ajustaram, além desses, o modelo de Bailey e Clutter para classificar sítios em povoamentos de Eucalyptus sp. no estado de São Paulo.

Dada a importância da classificação dos sítios florestais, objetivou-se neste trabalho testar diversos modelos estatísticos para ajuste de curvas de índice de sítio anamórficas e verificar se elas são satisfatórias para classificação de sítios em povoamentos de Pinus taeda na região de Caçador, no estado de Santa Catarina.

\section{MATERIAL E MÉTODOS}

\section{Descrição da área de estudo}

Para a realização deste estudo, os dados foram coletados em povoamentos de Pinus taeda da empresa Juliana Florestal Ltda., localizados na região de Caçador, SC. 
A área de estudo compreende, além de Caçador, os municípios de Macieira, Calmon, Timbó Grande, Lebon Régis, Rio das Antas e Santa Cecília, abrangendo uma área de 11.328,8 ha, dos quais $6.634,4$ ha são área plantada.

O clima predominante na região, segundo a classificação de Köeppen, é do tipo Cfb, também chamado de clima temperado úmido com verão temperado. A precipitação média anual fica entre $1.600 \mathrm{e}$ $1.800 \mathrm{~mm}$. A temperatura média anual é de $16,6^{\circ} \mathrm{C}$ e a umidade relativa do ar média é de $78,2 \%$.

A topografia é acidentada, intercalada por morros, colinas e chapadas. De acordo com CIRAM (2002), as classes predominantes de solos da área de estudo são Nitossolos, Neossolos e Cambissolos.

Os povoamentos foram implantadas com espaçamento inicial de $2 \times 2,5 \mathrm{~m}$, totalizando 2.000 árvores por hectare. De acordo com o regime de manejo adotado, os povoamentos sofrem quatro desbastes seletivo-sistemáticos nas idades de 8, 13, 16 e 20 anos, procedendo-se o corte raso, preferencialmente, aos 24 anos.

\section{Caracterização dos dados}

Para obtenção dos dados, foram medidas 691 parcelas permanentes de área fixa, formato retangular, com áreas de 500 e $625 \mathrm{~m}^{2}$. As parcelas foram instaladas para o inventário contínuo da empresa, contemplando as idades de 5, 6, 7, 8, 9, 10, 12, 13, 19, 20, 21, 22, 23, 24, 25, 26, 27 e 28 anos. Foram distribuídas de forma aleatória nos povoamentos para abranger a maior variabilidade possível de produtividade desses povoamentos.

Conforme recomendado por Fishwick (1975, citado por TONINI, 2000), o cálculo das alturas dominantes seguiu o princípio de Assmann, em que a altura dominante foi obtida tomando-se a média das alturas das 100 árvores mais grossas por hectare.

\section{Classificação de sítios}

Os sítios foram classificados por método direto, com base no crescimento em altura dominante dos povoamentos de Pinus taeda. Para isso, foram ajustados diversos modelos estatísticos (Tabela 1), empregando-se regressão linear e não linear para os pares de dados de idade (1) e $h_{\text {dom }}$ coletados.

Os modelos utilizados foram selecionados na literatura observando-se aqueles que obtiveram melhores resultados em trabalhos semelhantes, como Batista; Couto (1986), Machado et al. (1997), Oliveira et al. (1998), Scolforo; Machado (1988), Selle et al. (1994) e Tonini (2001).

Tabela 1. Modelos estatísticos testados para classificação de sítios em povoamentos de Pinus taeda L. na região de Caçador, SC.

Table 1. Statistical models tested for site classification of Pinus taeda L. plantations in the region of Caçador, Santa Catarina State, Brazil.

\begin{tabular}{lcc}
\hline $\mathrm{N}$ & Modelo & Autor \\
\hline 1 & $\ln h_{\text {dom }}=b_{0}+b_{1}\left(\frac{1}{I}\right)$ & Schumacher \\
2 & $\ln h_{d o m}=b_{0}+b_{1}\left(\frac{1}{I}\right)+b_{2}\left(\frac{1}{I^{2}}\right)$ & $\begin{array}{c}\text { Schumacher } \\
\text { modificado }\end{array}$ \\
3 & $\ln h_{\text {dom }}=\frac{\mathrm{I}^{2}}{b_{0}+b_{1} I+b_{2} I^{2}}$ & Prodan \\
4 & $\left.\ln h_{\text {dom }}=b_{0}+b_{1}\left(\frac{1}{I}\right)^{b_{2}}\right)$ & Bailey e Clutter \\
5 & $h_{\text {dom }}=b_{0}\left(1-e^{\left(b_{1} D\right.}\right)$ & Monomolecular \\
6 & $h_{\text {dom }}=b_{0}\left(1-e^{\left(b_{1} D\right.}\right)^{b_{2}}$ & Chapman e Richards \\
\hline
\end{tabular}

b0, b1, b2: coeficientes estimados pelo ajuste dos modelos estatísticos; $\mathrm{h}_{\mathrm{dom}}$ : altura dominante; I: idade; ln: logaritmo natural; $e$ : base do logaritmo natural.

O ajuste dos diferentes modelos estatísticos, apresentados na tabela 1, foi realizado com a utilização dos softwares MS Excel (2007) e STATISTICA 7. 
Os modelos lineares ou linearizáveis foram ajustados pelo método dos mínimos quadrados, enquanto os não lineares foram ajustados por regressão não linear, com a utilização do algoritmo matemático de Gauss e Newton, que atua minimizando a soma dos quadrados do erro.

Neste estudo, os modelos ajustados foram avaliados por meio das seguintes estatísticas de ajuste e precisão: análise gráfica de resíduos em porcentagem, coeficiente de determinação ajustado $\left(\mathrm{R}^{2}{ }_{\mathrm{aj}}\right.$ ) , erro padrão da estimativa (Syx) e erro padrão da estimativa em porcentagem (Syx\%).

Os modelos logarítmicos tiveram as estatísticas de ajuste e precisão transformadas para os valores originais da variável de interesse $h_{\text {dom }}$, ou seja, metros, com o auxílio do fator de correção de Meyer (Fm) para correção da discrepância logarítmica. Assim, as estatísticas de ajuste e precisão puderam ser comparadas entre modelos logarítmicos e não logarítmicos. O fator de correção de Meyer foi calculado como segue:

$$
\mathrm{Fm}=\mathrm{e}^{\left(0,5 * \mathrm{QM}_{\mathrm{m}} \mathrm{m}\right)}
$$

Em que: $e=$ base do logaritmo natural;

$\mathrm{QM}_{\text {erro }}=$ quadrado médio do erro. de local.

Após a seleção do modelo, empregou-se o método da curva guia para gerar as curvas de índice

A idade recomendada para a construção do conjunto de curvas deve estar em torno da idade de rotação da espécie em estudo (MACHADO et al., 1997). Como o corte raso das florestas de Pinus taeda da área de estudo ocorre somente a partir do $24^{\circ}$ ano, esta foi a idade adotada como idade de referência para a classificação dos sítios.

\section{Teste de anamorfismo}

O teste de anamorfismo consiste basicamente em verificar a existência de relação linear entre os índices de sítio e as alturas dominantes médias nas diferentes idades. No entanto, antes de verificar essa relação linear por meio de regressão linear simples, foi necessário encontrar o valor do índice de sítio correspondente a cada valor de $\mathrm{h}_{\mathrm{dom}}$, por meio da seguinte expressão matemática:

$$
I S=h_{d o m}\left(\frac{1-e^{\mathrm{D} 1 * I_{r e f}}}{1-e^{b 1+I}}\right)
$$

Em que: IS = índice de sítio;

$\mathrm{h}_{\mathrm{dom}}=$ altura dominante;

$\mathrm{I}_{\mathrm{ref}}=$ idade de referência;

$\mathrm{I}=$ idade para a qual a relação linear entre IS- $\mathrm{h}_{\mathrm{dom}}$ será ajustada;

$\mathrm{b}_{1}=$ coeficiente do modelo estatístico ajustado para classificação de sítios.

Posteriormente, a confiabilidade das curvas anamórficas foi testada, verificando-se a existência de relação linear entre os índices de sítio e as alturas dominantes médias nas diversas idades, por meio do ajuste do seguinte modelo estatístico:

$$
I S=b_{0}+b_{1} h_{d o m}
$$

Em que: IS = índice de sítio;

$\mathrm{h}_{\mathrm{dom}}=$ altura dominante média nas diferentes idades;

$\mathrm{b}_{0}$ e $\mathrm{b}_{1}=$ coeficientes estimados pelo ajuste do modelo.

O modelo linear simples foi ajustado para confirmar a hipótese de que há forte relação linear entre as variáveis envolvidas, expressando que o índice de sítio não depende da idade, mas sim da capacidade produtiva do local. 
Nesse caso, segundo Scolforo (2006), os resultados esperados são: coeficiente $b_{0}$ estatisticamente igual a zero e coeficiente $b_{1}$ estatisticamente maior que 1 nas idades menores do que a idade de referência, sendo tanto maior quanto menores forem as idades. $O$ coeficiente $b_{1}$, ainda, deve ser estatisticamente igual a um na idade de referência e menor que 1 nas idades acima desta.

Também foram avaliados os coeficientes de variação (CV) das alturas médias das árvores dominantes para cada idade em cada classe de sítio.

Para confirmação do anamorfismo, segundo Scolforo (2006), os coeficientes de variação devem apresentar valores semelhantes para as diversas idades de uma mesma classe de sítio.

\section{RESULTADOS E DISSCUSÃO}

\section{Classificação de sítios}

Os coeficientes e as estatísticas de diferentes modelos estatísticos testados são apresentados na tabela 2, além do fator de correção de Meyer para os modelos estatísticos logarítmicos.

Tabela 2. Coeficientes e estatísticas de ajuste de diferentes modelos para classificação de sítios de povoamentos de Pinus taeda L. na região de Caçador, SC.

Table 2. Coefficients and statistics of different models for site classification of Pinus taeda L. plantations in the region of Caçador, Santa Catarina State, Brazil.

\begin{tabular}{lccccccc}
\hline \multirow{2}{*}{ Modelos } & \multicolumn{9}{c}{ Coeficientes } & \multicolumn{3}{c}{ Estatísticas de ajuste e precisão } & \multirow{2}{*}{ Fm } \\
\cline { 2 - 7 } & $\mathbf{b}_{\mathbf{0}}$ & $\mathbf{b}_{\mathbf{1}}$ & $\mathbf{b}_{\mathbf{2}}$ & $\mathbf{R}_{\text {aj. }}^{2}$ & $\mathbf{S y x}$ & $\mathbf{S y x} \%$ & \\
\hline 1 & 3,746131 & $-8,170025$ & & 0,95575 & 2,2464 & 10,130 & 1,00500 \\
2 & 3,994067 & $-15,20456$ & 30,18212 & 0,96690 & 1,9429 & 8,7619 & 1,00362 \\
3 & $-3,97246$ & 1,985426 & 0,217730 & 0,96991 & 1,8525 & 8,3546 & \\
4 & 11,62756 & $-10,94890$ & 0,090219 & 0,97072 & 1,8266 & 8,2377 & 1,00329 \\
5 & 56,59182 & $-0,032165$ & & 0,96964 & 1,8608 & 8,3920 & \\
6 & 56,59022 & $-0,027551$ & 1,167473 & 0,96962 & 1,8615 & 8,3948 & \\
\hline
\end{tabular}

Todos os modelos apresentaram coeficientes de determinação ajustado maiores que 0,95 , indicando que mais de $95 \%$ da variação total da variável dependente $\left(\mathrm{h}_{\mathrm{dom}}\right)$ foi explicada pela regressão.

Os modelos 2, 3, 4, 5 e 6 apresentaram estatísticas de ajuste e precisão muito próximas. No entanto podem ser observadas algumas diferenças nos gráficos de dispersão de resíduos (Figura 1).

O modelo 1 foi o que apresentou as piores estatísticas de ajuste e precisão, além da distribuição de resíduos ter sido tendenciosa e resultar em superestimativa para os valores intermediários da variável dependente estimada $\left(\hat{\mathrm{Y}}_{\mathrm{j}}\right)$, conforme pode ser visto na figura 1 . Dentre os modelos com melhor desempenho, destacam-se os modelos 4, 5 e 6 . Apesar de apresentarem piores estatísticas de ajuste e precisão do que o modelo 4, os modelos 5 e 6 apresentaram ligeira superioridade na distribuição de resíduos (Figura 1). Quando comparados os modelos 5 e 6, verifica-se que há vantagem do modelo 5 sobre o modelo 6 , pois o modelo 5 descreve adequadamente a variação da altura dominante em função da idade utilizando apenas dois coeficientes, enquanto que o modelo 6 apresenta três.

Scolforo; Machado (1988a, 1988b), Selle et al. (1994), Tonini et al. (2001) e Ferreira et al. (2006) obtiveram os melhores ajustes para os modelos de Chapman e Richards e monomolecular entre os modelos testados pelos autores em estudos em povoamentos de Pinus taeda e Pinus elliottii na região Sul do Brasil.

As curvas de índice de sítio construídas a partir da equação selecionada (monomolecular) podem ser observadas na figura 2. Na tabela 3 são apresentados os limites inferior e superior das alturas dominantes, em metros, para cada uma das três classes de sítio e para todas as idades. Esses valores da tabela 3 correspondem às curvas que delimitam as classes de sítio apresentadas na figura 2 . De posse da tabela 3, é possível fazer a classificação de sítio de qualquer parcela pertencente à floresta de Pinus taeda da região de estudo conhecendo-se a sua idade $\mathrm{e} \mathrm{h}_{\mathrm{dom}}$.

Ferreira et al. (2006) encontraram valores semelhantes aos encontrados neste estudo para os limites das classes de sítios de uma floresta de Pinus taeda em Arapoti, PR. Entretanto Scolforo; Machado (1988a) encontraram uma maior amplitude dos valores de altura dominante nas diferentes 
idades. Isso se deve ao fato de que esses autores utilizaram dados de povoamentos de Pinus taeda de todo o estado do Paraná e Santa Catarina.

Modelo 1

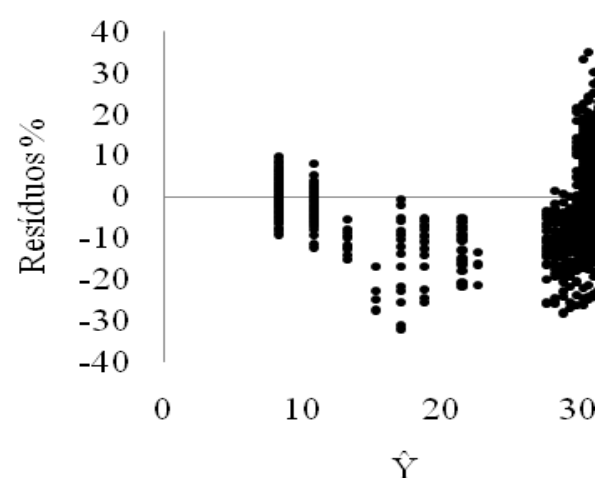

Modelo 3

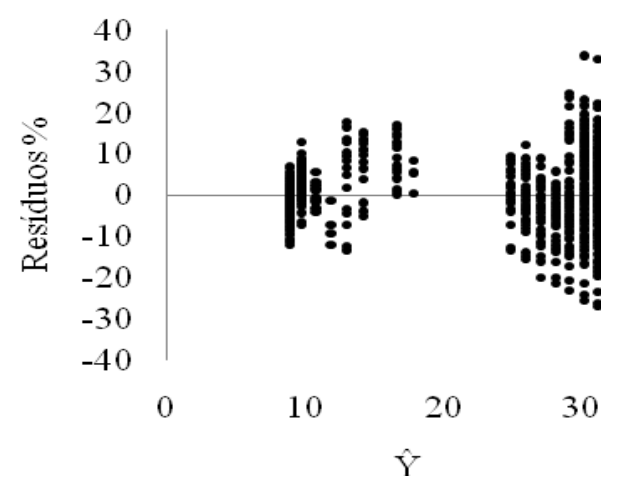

Modelo 5

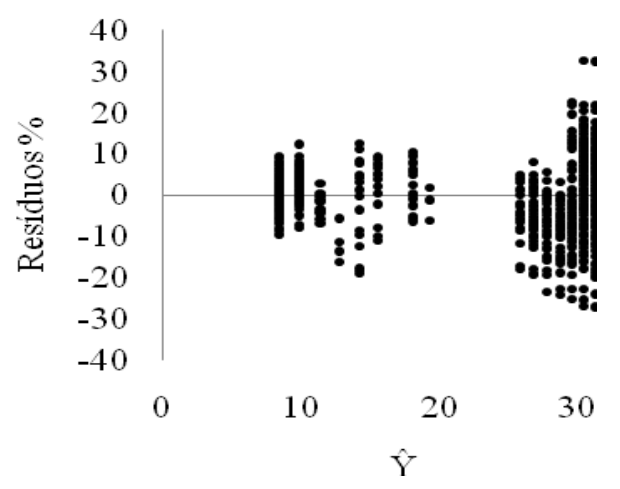

Modelo 2

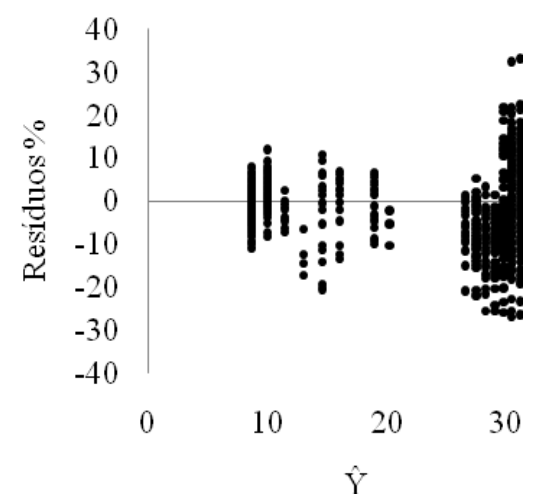

Modelo 4

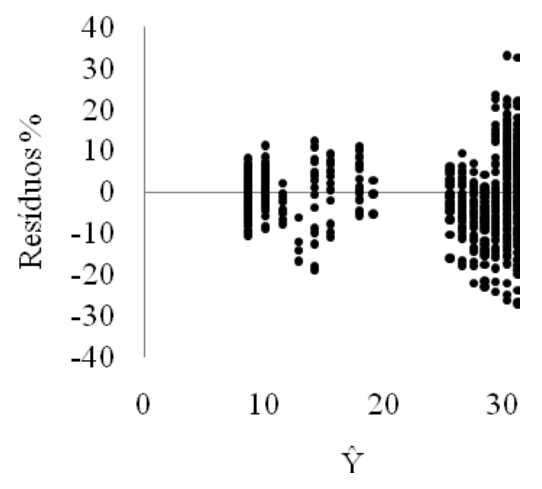

Modelo 6

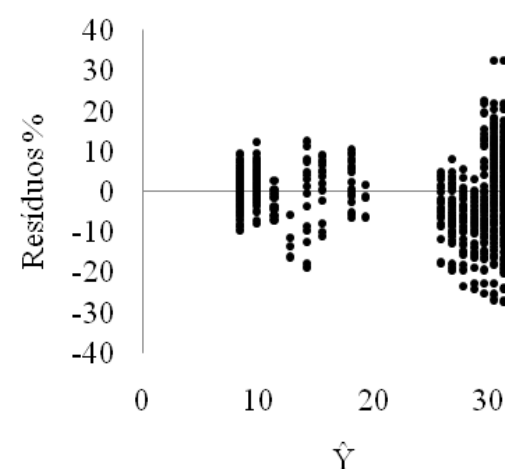

Figura 1. Gráficos de dispersão de resíduos (\%) em função da $\mathrm{h}_{\text {dom }}$ estimada para os modelos testados para classificação de sítios em povoamentos de Pinus taeda L. na região de Caçador, SC.

Figure 1. Residual distribution (\%) plotted against estimated dominant height for the mathematical models tested for site classification in Pinus taeda L. plantations in the region of Caçador, Santa Catarina State, Brazil. 


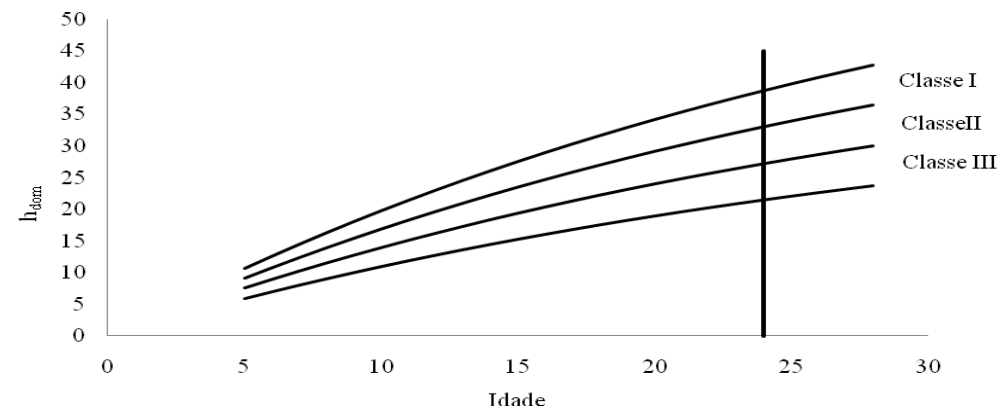

Figura 2. Curvas de índice de sítio para povoamentos de Pinus taeda L. na região de Caçador, SC.

Figure 2. Site index curves for Pinus taeda L. plantation in the region of Caçador, Santa Catarina State, Brazil.

Tabela 3. Limites das classes de sítio para todas as idades de povoamentos de Pinus taeda L. na região de Caçador, SC.

Table 3. Site classes limits for all ages of Pinus taeda L. plantations in the region of Caçador, Santa Catarina State, Brazil.

\begin{tabular}{lcccccc}
\hline \multirow{2}{*}{ Idade } & \multicolumn{2}{c}{ Classe I } & \multicolumn{2}{c}{ Classe II } & \multicolumn{2}{c}{ Classe III } \\
\cline { 2 - 7 } & LS & LI & LS & LI & LS & LI \\
\hline 5 & 10,7 & 9,1 & 9,1 & 7,5 & 7,5 & 5,9 \\
6 & 12,7 & 10,8 & 10,8 & 8,9 & 8,9 & 7,0 \\
7 & 14,5 & 12,4 & 12,4 & 10,2 & 10,2 & 8,0 \\
8 & 16,4 & 13,9 & 13,9 & 11,5 & 11,5 & 9,1 \\
9 & 18,1 & 15,4 & 15,4 & 12,7 & 12,7 & 10,0 \\
10 & 19,8 & 16,9 & 16,9 & 13,9 & 13,9 & 11,0 \\
11 & 21,5 & 18,3 & 18,3 & 15,1 & 15,1 & 11,9 \\
12 & 23,1 & 19,7 & 19,7 & 16,2 & 16,2 & 12,8 \\
13 & 24,7 & 21,0 & 21,0 & 17,3 & 17,3 & 13,6 \\
14 & 26,2 & 22,3 & 22,3 & 18,4 & 18,4 & 14,5 \\
15 & 27,6 & 23,5 & 23,5 & 19,4 & 19,4 & 15,3 \\
16 & 29,0 & 24,7 & 24,7 & 20,4 & 20,4 & 16,1 \\
17 & 30,4 & 25,9 & 25,9 & 21,3 & 21,3 & 16,8 \\
18 & 31,7 & 27,0 & 27,0 & 22,3 & 22,3 & 17,5 \\
19 & 33,0 & 28,1 & 28,1 & 23,2 & 23,2 & 18,3 \\
20 & 34,2 & 29,1 & 29,1 & 24,0 & 24,0 & 18,9 \\
21 & 35,4 & 30,1 & 30,1 & 24,9 & 24,9 & 19,6 \\
22 & 36,6 & 31,1 & 31,1 & 25,7 & 25,7 & 20,2 \\
23 & 37,7 & 32,1 & 32,1 & 26,5 & 26,5 & 20,9 \\
24 & 38,8 & 33,0 & 33,0 & 27,2 & 27,2 & 21,5 \\
25 & 39,9 & 33,9 & 33,9 & 28,0 & 28,0 & 22,1 \\
26 & 40,9 & 34,8 & 34,8 & 28,7 & 28,7 & 22,6 \\
27 & 41,9 & 35,6 & 35,6 & 29,4 & 29,4 & 23,2 \\
28 & 42,8 & 36,4 & 36,4 & 30,1 & 30,1 & 23,7 \\
\hline LS: & 23 & & & & &
\end{tabular}

LS: Limite Superior; LI: Limite Inferior.

\section{Teste de anamorfismo}

Na tabela 4 são apresentados os resultados dos ajustes do modelo linear do teste de anamorfismo, o qual relaciona o índice de sítio com a altura dominante nas diferentes idades, conforme descrito anteriormente na metodologia.

Segundo demonstrado na tabela 4 , os ajustes dos modelos estatísticos do teste de anamorfismo apresentaram valores para o coeficiente $b_{0}$ muito próximos de zero. Já o coeficiente $b_{1}$ foi maior que 1 nas menores idades, apresentando valores tanto maiores quanto menores fossem as idades. Verificou-se que $b_{1}$ foi igual a 1 na 
idade de referência e menor que 1 nas idades acima desta, sendo tanto menores quanto maiores fossem as idades. De acordo com os resultados encontrados, as curvas de índice de sítio seguem o padrão anamórfico.

Tabela 4. Resultados do ajuste dos modelos lineares para índice de sítio e altura dominante $\left(\mathrm{h}_{\mathrm{dom}}\right)$ do teste de anamorfismo para as curvas de índice de sítio de povoamentos de Pinus taeda L. na região de Caçador, SC.

Table 4. Results of the fitting of linear mathematical models for site index and dominant height $\left(\mathrm{h}_{\mathrm{dom}}\right)$ of the test of anamorphism for site index curves of Pinus taeda L. plantations, in the region of Caçador, Santa Catarina State, Brazil.

\begin{tabular}{|c|c|c|c|c|c|}
\hline Idade & & ientes & Idade & & entes \\
\hline 5 & $\begin{array}{l}\mathrm{b}_{0} \\
\mathrm{~b}_{1}\end{array}$ & $\begin{array}{l}3,90799 \mathrm{E}-14 \\
3,62075587\end{array}$ & 20 & $\begin{array}{l}\mathrm{b}_{0} \\
\mathrm{~b}_{1}\end{array}$ & $\begin{array}{c}3,55271 \mathrm{E}-15 \\
1,13373353\end{array}$ \\
\hline 6 & $\begin{array}{l}\mathrm{b}_{0} \\
\mathrm{~b}_{1}\end{array}$ & $\begin{array}{c}5,68434 \mathrm{E}-14 \\
3,06476099\end{array}$ & 21 & $\begin{array}{l}\mathrm{b}_{0} \\
\mathrm{~b}_{1}\end{array}$ & $\begin{array}{r}2,84217 \mathrm{E}-14 \\
1,09532772\end{array}$ \\
\hline 7 & $\begin{array}{l}\mathrm{b}_{0} \\
\mathrm{~b}_{1}\end{array}$ & $\begin{array}{l}1,42109 \mathrm{E}-14 \\
2,66803217\end{array}$ & 22 & $\begin{array}{l}b_{0} \\
b_{1}\end{array}$ & $\begin{array}{c}3,55271 \mathrm{E}-15 \\
1,06053859\end{array}$ \\
\hline 8 & $\begin{array}{l}\mathrm{b}_{0} \\
\mathrm{~b}_{1}\end{array}$ & $\begin{array}{c}-1,42109 \mathrm{E}-14 \\
2,37084417\end{array}$ & 23 & $\begin{array}{l}b_{0} \\
b_{1}\end{array}$ & $\begin{array}{c}0 \\
1,02889387\end{array}$ \\
\hline 9 & $\begin{array}{l}b_{0} \\
b_{1}\end{array}$ & $\begin{array}{c}-1,42109 \mathrm{E}-14 \\
2,14001622\end{array}$ & 24 & $\begin{array}{l}\mathrm{b}_{0} \\
\mathrm{~b}_{1}\end{array}$ & $\begin{array}{c}-7,1054 \mathrm{E}-15 \\
1\end{array}$ \\
\hline 10 & $\begin{array}{l}\mathrm{b}_{0} \\
\mathrm{~b}_{1}\end{array}$ & $\begin{array}{c}-1,77636 \mathrm{E}-14 \\
1,95563979\end{array}$ & 25 & $\begin{array}{l}\mathrm{b}_{0} \\
\mathrm{~b}_{1}\end{array}$ & $\begin{array}{c}1,42109 \mathrm{E}-14 \\
0,97352633\end{array}$ \\
\hline 12 & $\begin{array}{l}b_{0} \\
b_{1}\end{array}$ & $\begin{array}{c}3,55271 \mathrm{E}-15 \\
1,67978809\end{array}$ & 26 & $\begin{array}{l}\mathrm{b}_{0} \\
\mathrm{~b}_{1}\end{array}$ & $\begin{array}{c}0 \\
0,94919310\end{array}$ \\
\hline 13 & $\begin{array}{l}b_{0} \\
b_{1}\end{array}$ & $\begin{array}{c}-1,06581 \mathrm{E}-14 \\
1,57401933\end{array}$ & 27 & $\begin{array}{l}\mathrm{b}_{0} \\
\mathrm{~b}_{1}\end{array}$ & $\begin{array}{c}1,77636 \mathrm{E}-14 \\
0,92676195\end{array}$ \\
\hline 19 & $\begin{array}{l}b_{0} \\
b_{1}\end{array}$ & $\begin{array}{c}-3,55271 \mathrm{E}-15 \\
1,17632769\end{array}$ & 28 & $\begin{array}{l}\mathrm{b}_{0} \\
\mathrm{~b}_{1}\end{array}$ & $\begin{array}{r}-1,0658 \mathrm{E}-14 \\
0,90602858\end{array}$ \\
\hline
\end{tabular}

Scolforo; Machado (1988a) aplicaram o mesmo teste de anamorfismo para classificação de sítios em povoamentos de Pinus taeda nos estados do Paraná e Santa Catarina e confirmaram o anamorfismo das curvas construídas tal como ocorreu no presente trabalho.

Selle et al. (1994) fizeram verificação gráfica de alturas medidas em árvores nos povoamentos estudados sobre as curvas de índices de sítio anamórficas geradas para esses mesmos povoamentos, confirmando que as curvas de índice de sítio anamórficas descrevem bem o crescimento em $\mathrm{h}_{\mathrm{dom}}$ para Pinus taeda na região de Cambará do Sul, RS.

Cunha Neto et al. (1996) testaram o anamorfismo das curvas de índice de sítio geradas para Eucalyptus grandis e Eucalyptus urophylla na região de Luiz Antônio, SP, e concluíram que o desenvolvimento de ambas as espécies segue padrão anamórfico nos sítios estudados.

$\mathrm{O}$ modelo linear simples ajustado para o índice de sítio e a altura dominante $\left(\mathrm{h}_{\mathrm{dom}}\right)$ na idade de 24 anos é apresentado na figura 3. Observa-se que o coeficiente $b_{1}$ é igual a 1 , o qual indica a inclinação de $45^{\circ}$ com relação ao eixo das abscissas. Esse resultado reforça a confirmação do padrão anamórfico das curvas de índice de sítio geradas para povoamentos de Pinus taeda encontrado nesta pesquisa.

Scolforo (1992) também utilizou o método da diferença algébrica para gerar curvas de índice de sítio para povoamentos de Pinus caribaea sujeitos a desbastes, localizados no município de Agudos, SP. 
Para testar o anamorfismo das curvas de índice de sítio geradas, foram feitas representações gráficas do índice de sítio em relação à altura dominante em diferentes idades.

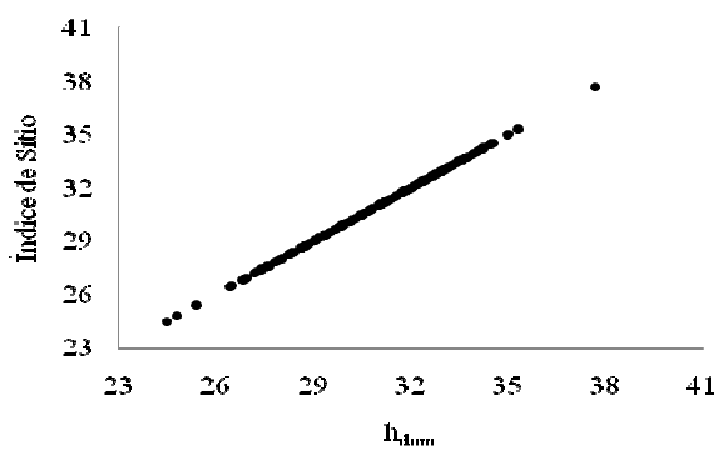

Figura 3. Índice de sítio em relação à $\mathrm{h}_{\mathrm{dom}}$ na idade de 24 anos para povoamentos Pinus taeda $\mathrm{L}$. na região de Caçador, SC.

Figure 3. Site index plotted against dominant height at the age of 24 years for Pinus taeda L. plantations in the region of Caçador, Santa Catarina State, Brazil.

Os coeficientes de variação (CV) das alturas médias das árvores dominantes para todas as idades referentes à classe de sítio I são apresentados na tabela 5.

Tabela 5. Coeficientes de variação para as diversas idades da classe de sítio I para povoamentos de Pinus taeda L. na região de Caçador, SC.

Table 5. Coefficients of variation for the several ages of the site class I of Pinus taeda L. plantations in the region of Caçador, Santa Catarina State, Brazil.

\begin{tabular}{lccccccccccc}
\hline \multicolumn{11}{c}{ Classe de sítio I } \\
\hline Idade & 5 & 6 & 9 & 10 & 12 & 23 & 24 & 25 & 26 & 27 & 28 \\
CV $(\%)$ & 8,83 & 5,50 & 3,21 & 3,79 & 3,26 & 6,73 & 5,01 & 4,94 & 5,40 & 5,70 & 3,61 \\
\hline
\end{tabular}

Os valores de coeficiente de variação encontrados comprovam o anamorfismo das curvas de índice de sítio construídas, visto que foram observados coeficientes de variação semelhantes para as diversas idades em uma mesma classe de sítio.

Scolforo (1992) fez a verificação dos coeficientes de variação das alturas médias das árvores dominantes por classe de sítio e idade, para povoamentos de Pinus caribaea no município de Agudos, SP. Encontrou coeficientes de variação semelhantes para as diversas idades de uma mesma classe de sítio, o que indica que as curvas de índice de sítio geradas seguiram o padrão anamórfico.

\section{CONCLUSÕES}

De acordo com os resultados, foi possível concluir que:

- O modelo monomolecular foi mais adequado para construção das curvas de índice de sítio para povoamentos de Pinus taeda na região de Caçador, SC.

- As curvas de índice de sítio para Pinus taeda na região de Caçador, SC, seguem o padrão anamórfico.

\section{REFERÊNCIAS}

BATISTA, J. L. F.; DO COUTO, H. T. Z. Escolha de modelos matemáticos para a construção de curvas de índice de sítio para florestas implantadas de Eucalyptus sp. no estado de São Paulo. IPEF, Piracicaba, n. 32, p. 33-42, abr. 1986. 
CIRAM. Mapa de solos unidade de planejamento regional meio-oeste catarinense UPR 2. Florianópolis: Epagri, 2002. 1 CD-ROM.

CUNHA NETO, F. R. da; SCOLFORO, J. R. S.; OLIVEIRA, A. D. de; CALEGÁRIO, N.; KANEGAE, H. K. J. Uso da diferença algébrica para construção de curvas de índice de sítio para Eucalyptus grandis e Eucalyptus urophilla na região de Luiz Antônio, SP. Revista Cerne, Lavras, v. 2, n. 2, p. 119-141, 1996.

DIAS, A. N.; LEITE, H. G.; NOGUEIRA, G. S.; RODRIGUES, F. L. Avaliação de métodos de ajuste de curvas de índice de local em povoamentos de eucaliptos desbastados. Revista Árvore, Viçosa, v. 29, n. 5, p. 741-747, set./out. 2005.

FERREIRA, M. Z.; GUIMARÃES, M. A. M.; SCOLFORO, J. R. S. Classificação de sítios florestais por meio de análise de tronco. Concórdia: Instituto Nacional de Tecnologia Agropecuária. 2006. 14 p.

FINGER, C. A. G. Fundamentos de biometria florestal. Santa Maria: UFSM, 2005. 286 p.

HAIR JR., J. F.; ANDERSON, R. E.; TATHAM, R. L.; BLACK, W. C. Análise multivariada de dados. 6. ed. Porto Alegre: Bookman, 2009. 688 p.

MACHADO, S. A.; OlIVEIRA, E. B. de; CARPANEZZI, A. A.; BARTOSZECK, A. C. P. S. Classificação de sítio para bracatingais na região metropolitana de Curitiba. Boletim de Pesquisa Florestal. Colombo, n. 35, p. 21-37, jul./dez. 1997.

OLIVEIRA, E. B. de; OLIVEIRA, Y. M. M. de; AHRENS, S.; MACHADO, S. A. Classificação de sítios para povoamentos de Pinus elliottii Engelm. no Sul do Brasil. Boletim de Pesquisa Florestal. Colombo, n. 37, p. 5-18, jul./dez. 1998.

SCOLFORO, J. R. S. Biometria florestal: modelos de crescimento e produção florestal. Lavras: FAEPE-UFLA, 2006. 393 p.

SCOLFORO, J. R. S. Curvas de índice de sítio para Pinus caribaea var. hondurensis. IPEF, Piracicaba, n. 45 , p. 40-47, jan./dez. 1992.

SCOLFORO, J. R. S.; MACHADO, S. A. Curvas de índice de sítio para plantações de Pinus elliottii nos estados do Paraná e Santa Catarina. Revista Floresta, Curitiba, v. 18, n. 12, p. 140-158 p, 1988.

Curvas de índice de sítio para plantações de Pinus taeda nos estados do Paraná e Santa Catarina. Revista Floresta, Curitiba, v. 18, n. 12, p. 159-173, 1988.

SELlE, G. L.; SCHNEIDER, P. R.; FINGER, C. A. G. Classificação de sítios para Pinus taeda L., através da altura dominante, para a região de Cambará do Sul, RS, Brasil. Ciência Florestal, Santa Maria, v. 4, n. 1, p. 77-95, 1994.

SHIMIZU, J. Y. Pinus na silvicultura brasileira. Curitiba: Embrapa, 2008. 223 p.

TONINI, H. Crescimento em altura de Pinus elliottii Engelm., em três unidades de mapeamento de solo, nas regiões da serra do sudeste e litoral, no estado do Rio Grande do Sul. 2000. 113 f. Dissertação (Mestrado em Engenharia Florestal) - Universidade Federal de Santa Maria, Santa Maria.

TONINI, H.; FINGER, C. A. G.; SCHNEIDER, P. R.; SPATHELF, P. Crescimento em altura de Pinus elliottii Engelm., na região de Piratini, Rio Grande do Sul, Brasil. Ciência Rural, Santa Maria, v. 31, n. 3, p. 417-423, jun. 2001. 